\title{
A simple method to deliver pharyngeal anesthesia in syndromic infants prior to awake insertion of the intubating laryngeal airway
}

\author{
Narasimhan Jagannathan, MD $\cdot$ Co T. Truong, MD
}

Received: 18 August 2010/Accepted: 16 September 2010/Published online: 28 September 2010

(C) Canadian Anesthesiologists' Society 2010

\section{To the Editor,}

Infants with a craniofacial abnormality who are potentially difficult to mask ventilate may benefit from awake placement of a supraglottic device to aid in tracheal intubation. ${ }^{1}$ Moreover, in some syndromic infants, severe upper airway obstruction may be present at birth, which often requires a mandibular advancement procedure or a tracheostomy. ${ }^{2}$ As these procedures require general anesthesia, clinicians may be hesitant to proceed with induction of anesthesia until upper airway obstruction is relieved. Options with the potential to alleviate airway obstruction prior to induction of anesthesia include: a change in position of the patient from supine to lateral or prone, placement of a naso-pharyngeal airway, ${ }^{3}$ or awake insertion of a supraglottic airway device. ${ }^{1}$ In this clinical scenario, the primary advantage of a supraglottic device is to relieve upper airway obstruction while providing a reliable conduit for tracheal intubation. Awake placement of the laryngeal mask airway $\left(\mathrm{LMA}^{\mathrm{TM}}\right.$ ) (LMA North America; San Diego, CA, USA) in the face of upper airway obstructions in syndromic infants is well tolerated without sedation or local anesthesia. ${ }^{1}$ However, the use of an air-Q intubating laryngeal airway (ILA ${ }^{\mathrm{TM}}$ ) (Cookgas LLC; St. Louis, MO, USA) for this same purpose can be more stimulating and therefore require some degree of pharyngeal anesthesia to minimize the risk of gagging upon placement. In our experience, the following features of the ILA make its insertion more stimulating in the awake infant when compared with the LMA: 1) the mask size of the ILA is wider and deeper than the LMA of equivalent

N. Jagannathan, MD $(\bowtie) \cdot C$. T. Truong, MD

Children's Memorial Hospital, Northwestern University's

Feinberg School of Medicine, Chicago, IL, USA

e-mail: simjag2000@yahoo.com size; 2) the airway tube of the ILA is wider, hyper-curved, and more rigid than the LMA (Figure A); and 3) the silicone-based reusable LMAs have a softer mask than the ILA. For these reasons, we find that infants do not tolerate awake placement of the ILA as well as they tolerate placement of the LMA. The primary advantage of the ILA for tracheal intubation is the capacity to place cuffed tracheal tubes with subsequent easy removal after tracheal intubation; ${ }^{4}$ therefore, we prefer use of the ILA over the LMA for purposes of tracheal intubation. The use of glossopharyngeal blocks in infants is effective to minimize the risk of gagging, ${ }^{5}$ but this method is invasive, and it can be more stressful and impractical in an awake child.

We describe a simple technique that we employ in our practice to provide pharyngeal anesthesia prior to awake ILA placement. Prior to inserting the ILA, we make use of a standard infant pacifier (Figure B) to deliver 2\% lidocaine jelly $20 \mathrm{mg} \cdot \mathrm{mL}^{-1}$ (AstraZeneca; Wilmington, DE, USA). Lidocaine jelly is injected into the nipple portion of an infant pacifier, and the pacifier is placed in the infant's mouth approximately 10 to $15 \mathrm{~min}$ prior to placement of the ILA. A maximum volume of approximately $5 \mathrm{~mL}$ can be accommodated within the nipple portion of an infant pacifier, this volume could be less based on the weight of the child, while adhering to the manufacturer's recommended maximal dose of $6 \mathrm{mg} \cdot \mathrm{kg}^{-1}$. ${ }^{\mathrm{A}}$ To achieve this dose, we mix lidocaine jelly with either a glucose solution or a surgical lubricant while remaining within these dosage guidelines. Several small perforations are then made in the nipple with a standard 20G needle to ensure pharyngeal spread upon infant sucking. Pharyngeal spread and systemic absorption of local anesthetic is governed by the

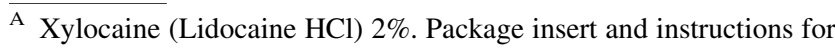
use. CAstraZeneca, Wilmington, DE 19850.
} 

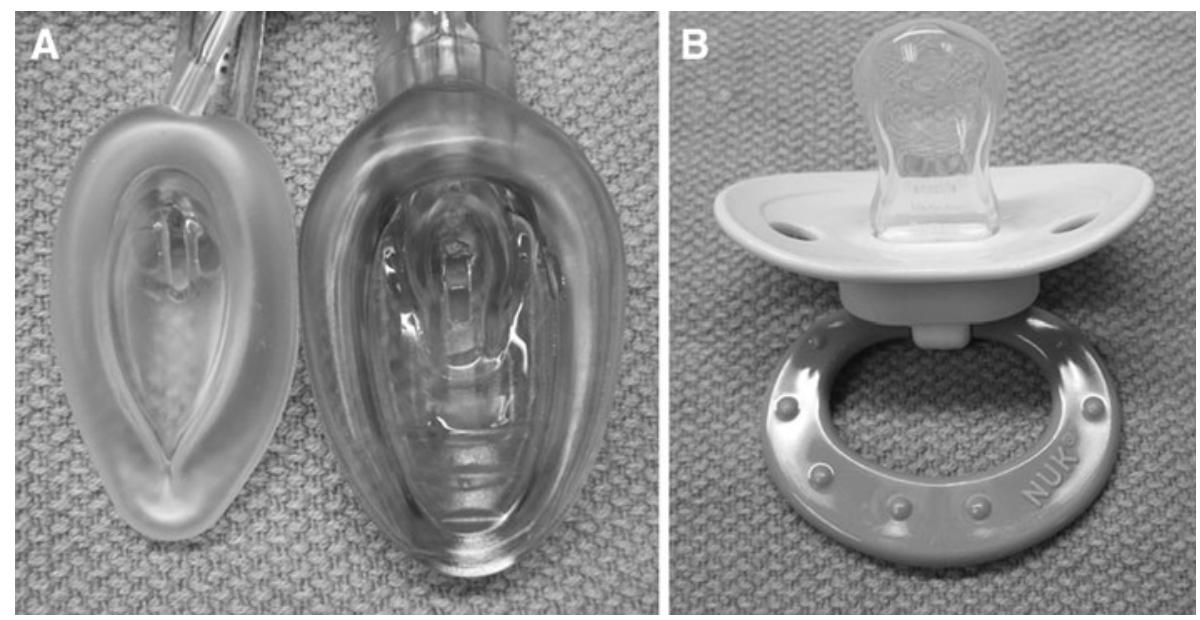

Figure Panel A: Anterior views of the mask bowls of the size 1 laryngeal mask airway (LMA) Unique (left) and the size 1 air-Q intubating laryngeal airway (ILA) (right). Notice the larger and widersized mask and airway tube of the ILA, which is also more rigid than the LMA. The larger dimensions of the ILA may contribute to a greater propensity for gagging upon placement in the awake infant.

degree of dryness of the pharyngeal mucosa, the nature of the infants sucking reflex, the viscosity and concentration of the final mixture, and the number of perforations created in the nipple. It should be recognized that premature neonates and small infants may have less protein binding of local anesthetic, therefore predisposing them to local anesthetic toxicity. In these patients, a dose of $3 \mathrm{mg} \cdot \mathrm{kg}^{-1}$ may suffice to provide anesthesia. However, clinicians should use their judgement when deciding on an adequate dose of local anesthetic required for an infant, and they should base their decision on the factors governing absorption of local anesthetic, the age of the patient, and the co-morbidities of the patient. The primary purpose of this technique is to provide anesthesia to the posterior tongue and upper esophagus, which can minimize the risk of gagging and thereby facilitate easier placement of the ILA in the awake infant.

We have used this technique in infants with PierreRobin and Treacher Collins syndromes with upper airway obstruction as a means to deliver pharyngeal spread of lidocaine when the ILA is inserted in the awake state. Placement of the ILA is then well tolerated and can potentially minimize the risk of gagging. Once inserted, upper airway obstruction is usually overcome and either induction of anesthesia or tracheal intubation can be performed according to the clinician's preference. An alternative approach for clinicians with less experience in airway management, e.g., neonatal intensive care unit staff or trainees, can be awake intubation by direct laryngoscopy with a bougie. The clinician should realize that exposure of the glottic opening may be difficult in severely micrognathic infants, and an alternative approach to secure the
Panel B: A standard infant pacifier. Lidocaine $2 \%$ jelly is injected within the nipple of the pacifier. Up to approximately $5 \mathrm{~mL}$ of total volume can be accommodated within this space. Several perforations in the nipple are then created with a needle to provide pharyngeal anesthesia when the infant begins sucking

airway may be necessary if direct laryngoscopy does not allow for tracheal intubation. Our method of delivering pharyngeal anesthesia may also assist these clinicians in minimizing gagging during awake intubation.

In summary, use of an infant pacifier is a simple noninvasive method to deliver pharyngeal anesthesia. This technique allows for better tolerance of the ILA when placed in the awake state, particularly in full-term infants who are vigorous and exhibit a strong gag reflex.

Disclosure The ILA/air- $\mathrm{Q}^{\mathrm{TM}}$ devices used were generously provided by the manufacturer (Cookgas LLC; St. Louis, MO, USA). None of the authors has any affiliations that may be perceived to be conflicts of interest with the submitted material.

\section{References}

1. Asai T, Nagata A, Shingu K. Awake tracheal intubation through the laryngeal mask in neonates with upper airway obstruction. Paediatr Anaesth 2008; 18: 77-80.

2. Nargozian $C$. The airway in patients with craniofacial abnormalities. Paediatric Anaesth 2004; 14: 53-9.

3. Holm-Knudsen R, Eriksen K, Rasmussen LS. Using a nasopharyngeal airway during fiberoptic intubation in small children with a difficult airway. Paediatr Anaesth 2005; 15: 839-45.

4. Jagannathan N, Roth AG, Sohn LE, Park TY, Amin S, Suresh S. The new air-Q intubating laryngeal airway for tracheal intubation in children with anticipated difficult airway: a case series. Paediatr Anaesth 2009; 19: 618-22.

5. Jagannathan N, Sohn LE, Suresh S. Glossopharyngeal nerve blocks for awake laryngeal mask airway insertion in an infant with Pierre-Robin syndrome: can a glidescope come to the rescue? Paediatr Anaesth 2009; 19: 189-90. 